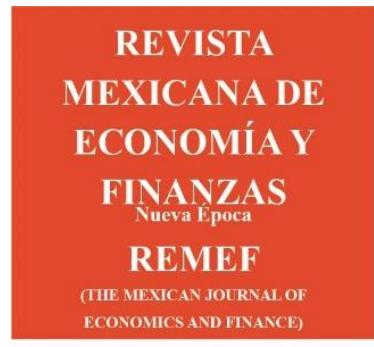

Revista Mexicana de Economía y Finanzas, Nueva Época

Volumen 16 Número 2, Abril - Junio 2021, pp. 1-21, e489

DOI: https://doi.org/10.21919/remef.v16i2.489

(Recibido: 14/abril/2020, aceptado: 1/octubre/2020, publicado: 12/enero/2021)

\title{
Ganancias cambiarias en empresas mexicanas y variables fundamentales y económicas
}

\author{
José Antonio Morales Castro ${ }^{1}$ - Instituto Politécnico Nacional, México \\ Francisco López-Herrera ${ }^{2}$ - Universidad Nacional Autónoma de México, México
}

El objetivo de este artículo de investigación es mostrar la evidencia de la influencia de variables económicas y fundamentales de empresa sobre las ganancias cambiarias derivadas de los descalces de moneda extranjera. Mediante modelos para datos en panel, se analizan datos trimestrales de 84 empresas mexicanas durante el periodo 1990-2018. Los resultados muestran que el dólar estadunidense y el PIB, así como seis de las variables fundamentales son significativas, cuatro de éstas últimas estudiadas por primera vez. Se recomienda considerar estas variables pues pueden ser útiles para orientar la toma de decisiones y el diseño de estrategias de operación, inversión y financiamiento para evitar pérdidas cambiarias. Obviamente, el alcance general de este estudio limita su aplicabilidad directa a casos particulares, haciendo necesario analizar la situación específica partiendo de los hallazgos aquí reportados. En México no se había estudiado el tema con la profundidad que se presenta en este documento, tampoco se habían analizado estas variables. Se concluye que las ganancias cambiarias reflejan aspectos relevantes de la economía, así como la importancia de las decisiones administrativas.

Clasificación JEL: F31, F36, F65, G32.

Palabras clave: ganancias (pérdidas) cambiarias, descalces cambiarios, descalces monetarios, variables

\section{Foreign Exchange Profits in Mexican Companies and Fundamental and Economic Variables}

The aim of this research paper is to show evidence of the influence of economic and fundamental company variables on the exchange rate gains derived from foreign currency mismatches. By means of panel data models, quarterly data of 84 Mexican companies are analyzed during the period 1990-2018. The results show that the US dollar and GDP, as well as six of the fundamental variables are significant, four of the latter studied for the first time. It is recommended to take in account these variables because they can be useful to guide the making decisions and the design of operation, investment and financing strategies to prevent foreign exchange losses. Obviously, the general scope of this study limits its direct applicability for particular cases, rendering necessary to analyze the specific situation based on the findings here reported. In Mexico, the subject had not been studied in the depth presented in this document, nor had these variables been analyzed. It is concluded that foreign exchange gains reflect relevant aspects of the economy, so as the importance of managerial decisions.

JEL Classification: F31, F36, F65, G32.

Keywords: Forex exchange profits (losses), currency mismatches, monetary mismatches, fundamental variables

\footnotetext{
1 Autor de correspondencia. correo electrónico: jmorales@ipn.mx; ORCID: http://orcid.org/0000-0001-5676-4745

2 correo electrónico: francisco_lopez_herrera@yahoo.com.mx; ORCID: http://orcid.org/0000-0003-2626-9246

* Sin fuente de financiamiento para el desarrollo de la investigación
} 


\section{Introducción}

Según Benavente, Johnson y Morandé (2003) y Cowan, Hansen y Herrera (2005), desde la década de 1990 se han presentado diversos episodios que depreciaron las monedas de los países emergentes y afectaron el desempeño financiero de empresas con pasivos en monedas extranjeras, a lo cual se ha denominado efecto de balance y que en algunos casos puede ser negativo. Serena y Sousa (2017) sostienen que dicho efecto no sólo incrementa los costos de la deuda y merman la rentabilidad de la empresa, también puede repercutir en los planes de inversión, en los de financiamiento y de producción e incluso, cuando se deteriora su solvencia, las puede llevar a dificultades financieras, incluyendo como caso extremo la quiebra. Durante la crisis de 1994 se devaluó drásticamente el peso mexicano, reflejándose en las empresas que tenían pasivos en moneda extranjera como un aumento notorio en sus niveles de apalancamiento y una reducción de su liquidez, aunque de acuerdo con Aguiar (2005) los efectos no fueron uniformes para todas las empresas.

Los descalces cambiarios se producen en las empresas con descalces (desajustes) monetarios que se dan si tienen activos en una moneda y pasivos en otra y/o sus ingresos son en una moneda diferente a la de sus egresos. Cuando los pasivos o egresos en moneda extranjera son mayores que los ingresos y/o activos en esa moneda, si se deprecia la moneda local se producen pérdidas cambiarias, pero si se aprecia se generan ganancias. Si los pasivos y/o los egresos en la moneda extranjera son menores que los activos y/o ingresos en la misma moneda, con la depreciación de la moneda nacional se obtienen ganancias cambiarias y con su apreciación se producen pérdidas. Morales et al. (2017) evidenciaron que el nivel de ventas extranjeras y la cantidad de deudas en moneda extranjera incrementan las ganancias cambiarias en las empresas de la Bolsa Mexicana de Valores durante el periodo 2011-2018.

En México se ha estudiado poco la influencia de las variables económicas y fundamentales de la empresa en las ganancias cambiarias como efecto de los descalces cambiarios registrados por las empresas. El presente trabajo contribuye al conocimiento de dichos efectos mostrando evidencia de la importancia que tienen en ellos variables económicas y fundamentales de empresa, incluyendo algunas de las cuales no se han analizado en la literatura previa hasta dónde llega nuestro conocimiento. Para tales efectos, en la sección siguiente se presenta una revisión de literatura pertinente. Posteriormente se dedica una sección a la descripción de las variables bajo estudio y del modelado para el análisis. En la sección del análisis de datos se presentan los resultados de las estimaciones, dedicando la sección final a las conclusiones.

\section{Revisión de la literatura}

Los efectos de los descalces cambiarios pueden llegar a debilitar la economía de los países emergentes, al respecto Céspedes, Chang y Velasco (2004) consideran que los tipos de cambio reales están vinculados con la deuda y los niveles de inversión de las empresas, reflejándose esta situación en la prima de riesgo país. Según este punto de vista, las grandes deudas denominadas en moneda 
extranjera hacen que una economía sea financieramente frágil, ya que las devaluaciones reales destruyen el patrimonio neto de las corporaciones y se vuelven contractivas y/o desestabilizadoras.

En relación con las crisis cambiarias, Krugman (1999) expone que se han utilizado diversos modelos para explicar la naturaleza de las crisis de mercados emergentes de 1997 a 1998, sin embargo, argumenta que el núcleo del problema radica en el sistema bancario, por lo que enfatiza que para explicar las crisis cambiarias se debe evaluar el papel de los balances de las empresas en lo que se refiere a su capacidad de inversión y generación de flujo de capital y su efecto en el tipo de cambio real. Díez y Ortiz (2001) citan a Krugman, quien describe que, a grandes rasgos, la crisis asiática de finales de los noventa se caracterizó por el deterioro en los balances de las empresas propiciado por el fuerte crecimiento de las deudas denominadas en moneda extranjera, lo que ha jugado un papel importante tanto en el origen de la crisis como en las dificultades a la hora de superarlas.

Aghion, Bacchetta y Banerjee (2000), afirman que una depreciación de la moneda conduce a las empresas a un aumento del pago de su deuda en moneda extranjera, y por lo tanto disminuyen sus ganancias, reduciéndose su capacidad de endeudamiento y de inversión. Si lo anterior se generaliza a la economía nacional, propicia efectos contractivos sobre la demanda de la moneda local contribuyendo así a una depreciación la moneda local. Morales (2020) analizó el efecto de las variaciones cambiarias en los índices de solvencia de las empresas del sector industrial de la Bolsa Mexicana de Valores durante el periodo de 2007 a 2017, corroborando que los niveles de solvencia no se deterioraron en las empresas que presentaron pérdidas cambiarias.

Forbes (2002) examina 12 depreciaciones importantes entre 1997 y 2000 y cómo afectaron a diferentes medidas de desempeño empresarial de una muestra de más de 13,500 compañías de todo el mundo. Sus resultados sugieren que en el año posterior a las depreciaciones las empresas tienen un crecimiento significativamente mayor en la capitalización de mercado, pero un crecimiento significativamente menor en el ingreso neto (cuando se mide en moneda local). Las empresas con una mayor proporción de ventas en el extranjero tienen un rendimiento significativamente mejor después de las depreciaciones, las que tienen índices de endeudamiento más altos tienden a tener un menor crecimiento del ingreso neto, pero no existe relación solida entre la exposición a la deuda y las otras variables de desempeño.

La mayoría de los estudios se han enfocado principalmente a analizar los efectos de las devaluaciones de la moneda en el desempeño de las empresas, y exposición a riesgos, entre los cuales se encuentran las investigaciones de Allayannis, Brown y Klapper (2003), en la que analizan un grupo de empresas del este de Asia examinando los factores que inciden en la elección de deuda en moneda extranjera. Encontraron que el menor costo (percibido) de la deuda en moneda extranjera, así como la necesidad de acceder a mercados de capital extranjero más profundos, explican consistentemente el tipo de deuda utilizada. Contrario a las conclusiones de investigaciones previas, no encontraron evidencia que sugiera que la deuda en moneda extranjera no cubierta era la causa principal del bajo desempeño durante las crisis cambiarias. Morales (2019) analizó la volatilidad de los precios de las acciones de las empresas del sector de consumo discrecional de la Bolsa Mexicana de Valores durante los ciclos de volatilidad cambiaria en el periodo 2011-2018, y encontró que no hubo alta volatilidad en los ciclos de depreciación de la moneda nacional.

Shapiro (1984) analiza el efecto de los impuestos en los costos de financiamiento en moneda extranjera, ofreciendo evidencia de la importancia de las tasas impositivas y de los efectos fiscales 
para las decisiones de financiamiento a largo plazo, siendo determinantes significativos de la elección, incluso de la moneda extranjera puesto que las tasas impositivas varían entre los países y, naturalmente, con ellas el costo del financiamiento en el extranjero.

Los descalces entre pasivos y activos en moneda extranjera obedecen a distintas causas, señala Chow (2015) que la deuda corporativa negociada en los mercados emergentes aumentó considerablemente en los últimos años, respaldada por las bajas tasas de interés y el fácil acceso a los mercados mundiales de capital, que las firmas de esos países han tenido, lo cual explica en parte los altos niveles de apalancamiento en moneda extranjera y por consiguiente un descalce monetario. Nuñez, Perrotini y López-Herrera (2018) muestran que a partir de 2009 el mercado de bonos corporativos internacionales se convirtió en una fuente de financiamiento importante para las empresas latinoamericanas, lo cual atribuyen en parte a las mejoras en el gobierno corporativo de esas empresa, así como a las bajas tasas de interés establecidas por las autoridades monetarias de los diferentes países como respuesta a la política de flexibilización aplicada por la Reserva Federal de los Estados Unidos para superar los efectos de la crisis financiera de 2008.

Al respecto, Caballero, Panizza y Powel (2014) sugieren que la profundización originada en la integración financiera mundial contribuyó a la expansión del crédito para las empresas de la región latinoamericana y del Caribe pero que, a medida que las tasas de Estados Unidos se normalizaran, era probable que la demanda de los inversionistas regionales disminuyese y, en algunos casos, las monedas se depreciaran rápidamente. Los descalces monetarios en los balances de los bancos, o de las empresas que han emitido instrumentos de deuda en los mercados internacionales, pueden tener efectos colaterales en los sistemas financieros nacionales, pues los balances corporativos se deterioran cuando se deprecia la moneda local, pudiendo incluso generar problemas de liquidez para las empresas endeudas en la moneda extranjera que se aprecia.

En los últimos 30 años las economías emergentes han protagonizado episodios de la depreciación de sus monedas y por ende la afectación a sus empresas, Chui, Kuruc y Turner (2016) explican que las empresas de las economías emergentes en conjunto se volvieron más rentables que las empresas de las economías avanzadas. En promedio, el coeficiente de cobertura de intereses ha disminuido significativamente a pesar de que las tasas de interés en los mercados globales han disminuido, las empresas de las economías emergentes enfrentan un aumento de los desajustes monetarios en balances más débiles. Con datos de alrededor de 280 empresas, Chui et al. (2016) muestran que los préstamos en moneda extranjera no han sido igualados con la moneda de sus ganancias y sugieren que el sector corporativo se hizo más vulnerable a choques monetarios y de tasas de interés.

Chui, Fender y Sushko (2014) argumentan que las empresas en las economías emergentes han incrementado los créditos contratados en moneda extranjera, y con ellos su apalancamiento, para aprovechar de que las condiciones financieras globales brindan facilidades inusuales; sin embargo, las choques en las tasas de interés y/o el tipo de cambio podrían generar bucles de retroalimentación perjudiciales, a menos de que estas posiciones estén adecuadamente cubiertas. Señalan que algunos episodios de depreciación de la moneda, por ejemplo, como el caso de Ucrania, hacen recordar cuán abruptamente pueden deteriorar las métricas de sostenibilidad de la deuda 
cuando existen riesgos geopolíticos que inflan el valor en moneda local de los pasivos en moneda extranjera.

Chui, Kuruc y Turner (2016) analizan el notable crecimiento del endeudamiento corporativo de las economías emergentes en los mercados globales de bonos en dólares, ya que un periodo muy largo de bajas tasa de interés a largo plazo ha permitido a las empresas de esas economías un financiamiento más barato. Algunas empresas han ampliado el alcance global de sus operaciones mediante un extraordinario aumento de su deuda total, que ha estado acompañado de una gran y amplia disminución en 2015 de su rentabilidad en comparación con las empresas de las economías avanzadas. Por su exposición a los altibajos de la paridad respecto del dólar, esas empresas tienen balances más débiles ante la presencia de descalces monetarios.

Du y Schreger (2016) analizaron datos de Brasil, Colombia, Hungría, Indonesia, Israel, Malasia, México, Perú, Polonia, Rusia, Sudáfrica, Corea del Sur, Tailandia y Turquía, encontrando que una mayor dependencia del financiamiento corporativo en moneda extranjera se asocia con un mayor riesgo de incumplimiento soberano, sobre todo porque generalmente los corporativos siguen dependiendo de la deuda en moneda extranjera, incluso cuando los gobiernos soberanos se han movido rápidamente hacia los préstamos en moneda local. Kim, Tesar y Zhang (2015) estudiaron datos en el nivel de empresa, encontrando evidencias de que las tenencias de deuda en moneda extranjera afectaron negativamente el desempeño económico de las pequeñas empresas durante la crisis de 1997-1998. Las pequeñas empresas con más deuda extranjera a corto plazo tenían más probabilidad de declararse en bancarrota porque experimentaron mayores descensos en las ventas.

También existe una serie de estudios que ha analizado los factores que influyen en el uso de deudas en moneda extranjera, entre ellos el de Keysriyeli, Özmen y Yigit. (2011) quienes señalan que tanto factores del sector específico de actividad económica (tangibilidad, razón de apalancamiento, participación de exportación, por ejemplo), como variables macroeconómicas (inflación, variación del tipo de cambio real, déficit público y confianza), son importantes para explicar la dolarización de pasivos de los sectores corporativos de Turquía, y consideran que las depreciaciones tienen efectos contractivos para las inversiones y ganancias de los sectores con mayores pasivos dolarizados.

Bae, Kim y Kwon (2018) encontraron que los determinantes de la deuda en moneda extranjera de las empresas coreanas se ven afectados principalmente por los incentivos del prestatario, la rentabilidad operativa y los gastos de depreciación que reflejan las necesidades de capital del prestatario; así como por la proporción de activos tangibles, el tamaño de la empresa, el crecimiento de los activos y la proporción de las ventas de exportación, la cual es indicador fundamentalmente que los prestamistas extranjeros usan para evaluar el valor potencial de las garantías. Estos factores están en línea con la literatura que sostiene que el índice de exportación de una empresa está significativamente relacionado con el uso de la deuda en moneda extranjera, evidencia que respalda el papel de cobertura. Bae, Kwon y Lee (2011) estudiaron una muestra de empresas coreanas de la industria manufacturera, encontrando que de 1998 a 2005 se presentaron asimetrías significativas en la exposición cambiaria, caracterizadas porque el índice de exportación y el índice de deuda denominada en dólares están significativamente relacionados con la exposición asimétrica: las exportaciones con signo negativo y el nivel de deudas en dólares con signo positivo. Además, mostraron que las empresas responden a diferentes determinantes de la exposición cambiaria asimétrica en función de sus giros operativos de exportación y/o tipo de financiamiento de deuda externa utilizada. 
Al estudiar el comportamiento entre 1971 y 1998 de un grupo de empresas estadounidenses, Frank y Goyal (2003) encontraron evidencias de que el financiamiento externo es muy utilizado, contradiciendo a la teoría del orden jerárquico (pecking order) que postula que las compañías priorizan sus fuentes de financiamiento, prefiriendo en primer lugar financiamiento de fuentes internas, después deuda y finalmente la emisión accionaria.

Podría ser que las empresas mexicanas tampoco se comporten de acuerdo con la teoría pecking order. Hernández y Ríos (2012) encontraron que de 2000 a 2009 las empresas del sector de alimentos tenían la composición de activos como el principal determinante de su estructura financiera, probablemente porque sus activos pueden servir como colaterales al momento de emitir deuda. Por su parte, García, García y Domenge (2011) sostienen que las empresas en México se endeudan más cuando son más grandes. En ambos estudios se afirma que las empresas en México prefieren deuda a fuentes internas.

Desai, Foley y Forbes (2007) encontraron diferencias en la respuesta de las empresas multinacionales y estadunidenses a las fuertes depreciaciones observadas en el periodo de 1991 a1999, las primeras expandieron sus ventas, los activos y la inversión, mientras que en las compañías locales se observaron disminuciones o cambios marginales. Consideran que los resultados de estos patrones no son únicamente consecuencia de oportunidades de inversión diferenciadas que surgen de los mercados de productos, sino, también de elementos que se ubican en la diferente capacidad para superar las restricciones financieras. También se debe considerar que las filiales de las multinacionales afiliadas pueden recibir infusiones de capital de sus matrices después de la depreciación, en tanto que las empresas locales con mayor exposición financiera experimentan las mayores reducciones de inversión.

Entre los estudios sobre la influencia de las variables macroeconómicas en la cuantía de las pérdidas cambiarías empresariales, se encuentra el de Bonono, Martins y Pinto (2003) quienes evaluaron corporaciones no financieras brasileñas con información contable anual por el período de 1990 a 2002, estudiando las interacciones de las condiciones macroeconómicas y su influencia en las estructuras de la deuda y la cantidad de inversión en los episodios de cambio en el nivel del riesgo cambiario. Encontraron evidencias de que las empresas con más deudas en moneda extranjera tienden invertir menos durante la devaluación de su moneda y que las devaluaciones tienen un impacto negativo importante, tanto en los flujos de efectivo como en las ventas de las empresas endeudadas.

Brown, Ongena y Yesin (2011) estudiaron los determinantes del nivel de endeudamiento en moneda extranjera de las pequeñas empresas de 25 países durante 2002-2005. Sus resultados sugieren que el endeudamiento en moneda extranjera de las pequeñas empresas es mucho más fuerte en relación con las características de la empresa, es decir, los ingresos en moneda extranjera, que, con las condiciones macroeconómicas, como es el caso de las diferencias de tasas de interés. Además, estos autores sugieren que los clientes minoristas que toman préstamos en moneda extranjera tienen más habilidades de lo que comúnmente se piensa para soportar los riesgos monetarios correspondientes.

Luca y Petrova (2008) analizan la profundidad del uso de moneda extranjera en las actividades crediticias de los bancos de 21 economías en transición durante 1990-2003. Sus 
resultados sugieren que los bancos expuestos al riesgo cambiario por el uso de pasivos, en dólares de fuentes nacionales y externas, transfieren este riesgo a las empresas mediante préstamos en dólares. Generalmente estos préstamos exceden las coberturas naturales de las empresas compensadas por los flujos de efectivo correlacionados positivamente con el tipo de cambio. Consecuentemente, los bancos disminuyen su exposición directa al riesgo cambiario, pero no ocurre lo mismo con las empresas no financieras porque generalmente no se protegen contra el riesgo cambiario, incluso en presencia de mercados forward de divisas profundos y líquidos.

Durante los años noventa se produjeron crisis cambiarías tanto en Europa como en Latinoamérica (el efecto tequila en México), en Asia (la gripe asiática, en Indonesia, Corea, Malasia, Filipinas y Tailandia), las cuales impactaron en gran medida a las economías emergentes, sus procesos de producción y en la regulación fiscal que se orientaron para rescatar el sector financiero excesivamente frágil, (Díez y Ortiz, 2001).

Como hemos visto, varios autores demuestran que durante los últimos 20 años las condiciones crediticias en los mercados financieros internacionales han favorecido el aumento del uso de deudas en moneda extranjera, lo cual acentúa el riesgo de sufrir descalces monetarios cuando las monedas locales se deprecian, siendo más vulnerables los países emergentes. Algunos estudios de otros países ofrecen evidencia de que las variables macroeconómicas y algunos indicadores específicos de las empresas influyen en los descalces cambiarios. Además, el deterioro de los balances de las empresas con descalces monetarios durante una depreciación de las monedas locales puede tener efectos colaterales en los sistemas financieros nacionales.

\section{Datos y aspectos metodológicos}

La pregunta de investigación que se plantea para el análisis econométrico que se presenta más adelante es: ¿cuáles variables económicas y fundamentales de la empresa tienen impacto en las ganancias cambiarias de las empresas mexicanas vía los descalces cambiarios?

De Economatica y de la Bolsa Mexicana de Valores se obtuvieron, en dólares, el balance general y el estado de resultados de las 84 empresas estudiadas: 14 del sector productos de consumo no básico, 24 del sector industrial, 17 del sector materiales, 16 del sector de consumo frecuente, 4 del sector salud y 9 del sector telecomunicaciones, véase anexo $1 .^{3}$ Del INEGI se obtuvo el Producto Interno Bruto (PIB) y del Banco de México el precio del dólar y el precio de la mezcla mexicana de petróleo. Todos los datos son trimestrales, pero no todas las empresas estudiadas cotizaron durante todo el periodo así que el número de observaciones difiere, las más amplias cubren del primer trimestre de 1990 al cuarto de 2018.

El análisis que se presenta en la sección posterior se llevó a cabo para determinar qué variables internas de la empresa y macroeconómicas explican el saldo cambiario, el cual refleja las ganancias (con signo positivo) y pérdidas cambiarias (con signo negativo) provenientes de la tenencia de pasivos y activos en moneda extranjera y de ingresos y egresos en moneda extranjera. La norma contable señala que a la fecha de cierre de los estados financieros presentan saldos de partidas

\footnotetext{
${ }^{3}$ Se excluye a las empresas del sector financiero porque su financiamiento lo obtienen principalmente de ahorradores nacionales, es decir, en la moneda local, por lo que no tienen un descalce monetario que las haga vulnerables, como las empresas no financieras, en los periodos de depreciación de las monedas locales.
} 
monetarias (cuentas por cobrar y cuentas por pagar) derivadas de transacciones en moneda extranjera y que estén denominadas en moneda extranjera deben convertirse al tipo de cambio con el que pudiera haberse realizado (cobrado o pagado). De estos procedimientos surge diferencias en cambios que deben reconocerse como ingreso o gasto en la utilidad o pérdida neta del estado de resultados integral en el período que se origina. Las NIF señalan que para determinar la utilidad o pérdida cambiaría debe utilizarse el tipo de cambio con el que pudiera haberse realizado (cobrado o pagado). En México se utiliza el tipo de cambio establecido por el Banco de México, denominado dólar fix, que se publica en el Diario Oficial de la Federación.

Como variables independientes, se escogieron el PIB, el precio del petróleo, el tipo de cambio peso-dólar, la inversión en activos intangibles y las razones financieras que miden la inversión en inmuebles, planta, equipo y capital de trabajo. Las variables macroeconómicas incluidas son las que frecuentemente han sido analizadas en otros estudios sobre los efectos de balance, como es el artículo de Bonono, Martins y Pinto (2003), y sobre todo los estudios que han incluido variables macroeconómicas en la determinación de la estructura de la deuda de moneda extranjera, entre los que se encuentran Krugman (1999), Chui, Fender y Sushko (2014) y Desai, Foley y Forbes (2007) y Keysriyeli, Özmen y Yigit. (2011).

Con los datos de los estados financieros se construyeron las variables internas de las empresas estudiadas, el detalle de esas variables se puede ver en el Cuadro 1.

\section{Cuadro 1. Variables utilizadas en este estudio}

\begin{tabular}{|c|c|c|}
\hline Variable & Construcción & Descripción \\
\hline $\begin{array}{l}\text { SCUN* } \\
\text { Ganancia } \\
\text { (pérdida) } \\
\text { derivadas del } \\
\text { saldo cambiario }\end{array}$ & $\begin{array}{l}\text { Saldo cambiario } \div \\
\text { Utilidad Neta }\end{array}$ & $\begin{array}{l}\text { El signo negativo indica perdidas cambiarias, el positivo } \\
\text { ganancias. Captura el efecto del descalce cambiario en las } \\
\text { ganancias totales. }\end{array}$ \\
\hline $\begin{array}{l}(\mathrm{AC}-\mathrm{INV}) / \mathrm{PC} \\
\text { Prueba acida }\end{array}$ & $\begin{array}{l}\text { Activo circulante } \\
\text { - inventario } \div \\
\text { Pasivo circulante }\end{array}$ & $\begin{array}{l}\text { Mide la capacidad de solventar las deudas de corto plazo con } \\
\text { los activos cuasi líquidos. }\end{array}$ \\
\hline $\begin{array}{l}\text { AC/AT } \\
\text { Inversión } \\
\text { Activos } \\
\text { corrientes }\end{array}$ & $\begin{array}{l}\text { Activos } \\
\text { corrientes } \\
\text { Activo total }\end{array}$ & Mide las inversiones de corto plazo de la inversión total. \\
\hline $\begin{array}{l}\text { AI } \\
\text { Inversión en } \\
\text { activos } \\
\text { intangibles }\end{array}$ & $\begin{array}{l}\text { Activos } \\
\text { intangibles } \quad \div \\
\text { Activo Total }\end{array}$ & $\begin{array}{l}\text { Mide la cantidad de inversión en patentes y marcas, del } \\
\text { total de inversiones. }\end{array}$ \\
\hline $\begin{array}{l}\text { AF/Act_Inv } \\
\text { Inversión a largo } \\
\text { plazo }\end{array}$ & $\begin{array}{l}\text { Inmuebles, } \\
\text { Planta y Equipo } \div \\
\text { Actividades } \\
\text { Inversión }\end{array}$ & $\begin{array}{l}\text { Cuantifica la proporción de la inversión de la inversión que } \\
\text { se hizo en inmuebles, planta y equipo. }\end{array}$ \\
\hline
\end{tabular}




\begin{tabular}{|c|c|c|}
\hline Variable & Construcción & Descripción \\
\hline $\begin{array}{l}\text { CTN } \\
\text { Capital de } \\
\text { trabajo }\end{array}$ & $\begin{array}{l}\text { Capital } \text { Trabajo } \\
\text { Neto } \div \text { Pasivo } \\
\text { Circulante } \\
\end{array}$ & $\begin{array}{l}\text { Mide las inversiones de corto plazo con relación a las } \\
\text { deudas de corto plazo. }\end{array}$ \\
\hline $\begin{array}{l}\text { CETES } \\
\text { Certificados } \\
\text { Tesorería } \\
\text { Federación }\end{array}$ & $\begin{array}{l}\text { Tasa de interés } \\
\text { del instrumento } \\
\text { considerado líder } \\
\text { en México }\end{array}$ & $\begin{array}{l}\text { Registra la tasa de interés que paga el gobierno de México } \\
\text { por financiarse en corto plazo. }\end{array}$ \\
\hline $\begin{array}{l}\text { DME/AT } \\
\text { Deudas } \\
\text { Moneda } \\
\text { Extranjera }\end{array}$ & $\begin{array}{l}\text { Deuda Moneda } \\
\text { Extranjera } \div \\
\text { Activo Total }\end{array}$ & $\begin{array}{l}\text { Porcentaje de activos que han sido financiados en moneda } \\
\text { extranjera. }\end{array}$ \\
\hline $\begin{array}{l}\text { DME/PT } \\
\text { Deudas } \\
\text { Moneda } \\
\text { Extranjera }\end{array}$ & $\begin{array}{l}\text { Deuda Moneda } \\
\text { Extranjera } \div \\
\text { Pasivo Total }\end{array}$ & $\begin{array}{l}\text { Identifica el porcentaje de deuda en moneda extranjera del } \\
\text { total de pasivos. }\end{array}$ \\
\hline Dólar & $\begin{array}{l}\text { Dólar Fix, } \\
\text { publicado por el } \\
\text { Banco de México }\end{array}$ & Precio del dólar en pesos mexicanos. \\
\hline $\begin{array}{l}\text { E/PC } \\
\text { Liquidez }\end{array}$ & $\begin{array}{lr}\text { Efectivo } \quad \text { y } \\
\text { Equivalentes } \quad \div \\
\text { Pasivo Circulante } \\
\end{array}$ & $\begin{array}{l}\text { Mide las veces que se puede pagar las deudas de corto } \\
\text { plazo con el efectivo disponible. }\end{array}$ \\
\hline $\begin{array}{l}\text { FA/TF } \\
\text { Financiamiento } \\
\text { Ajeno }\end{array}$ & $\begin{array}{l}\text { Financiamiento } \\
\text { Ajeno } \div \text { Total } \\
\text { Financiamiento }\end{array}$ & $\begin{array}{l}\text { Refleja la cantidad de financiamiento que proviene por } \\
\text { deuda del total de financiamiento. }\end{array}$ \\
\hline $\begin{array}{l}\text { FP/TF } \\
\text { Financiamiento } \\
\text { Propio }\end{array}$ & $\begin{array}{l}\text { Financiamiento } \\
\text { Propio } \div \text { Total } \\
\text { Financiamiento }\end{array}$ & $\begin{array}{l}\text { Refleja la cantidad de financiamiento que proviene de los } \\
\text { socios del total de financiamiento. }\end{array}$ \\
\hline $\begin{array}{l}\text { FCT/VN } \\
\text { Flujo de capital } \\
\text { de trabajo }\end{array}$ & $\begin{array}{ll}\text { Flujo } & \text { capital } \\
\text { trabajo } \div \text { Ventas } \\
\text { Netas }\end{array}$ & $\begin{array}{l}\text { Representa las inversiones en activos circulantes } \\
\text { aportadas por los socios en términos de ventas. }\end{array}$ \\
\hline $\begin{array}{l}\text { FRN/VN } \\
\text { Flujo de } \\
\text { resultado neto }\end{array}$ & $\begin{array}{lr}\text { Flujo } & \text { resultado } \\
\text { neto } \div \text { Ventas } \\
\text { Netas }\end{array}$ & $\begin{array}{l}\text { Mide la utilidad neta junto con las partidas virtuales en } \\
\text { términos de ventas. }\end{array}$ \\
\hline $\begin{array}{l}\text { AF/AT } \\
\text { Activos largo } \\
\text { plazo }\end{array}$ & $\begin{array}{l}\text { Inmuebles, } \\
\text { Planta y Equipo } \div \\
\text { Activo Total }\end{array}$ & $\begin{array}{l}\text { Es la cantidad de inversiones en activos fijos del total de } \\
\text { inversión expresado en veces. }\end{array}$ \\
\hline $\begin{array}{l}\text { I/RGO } \\
\text { Intereses } \\
\text { pagados }\end{array}$ & $\begin{array}{l}\text { Interés Pagado } \div \\
\text { Recursos } \\
\text { Generados } \\
\text { Operación }\end{array}$ & $\begin{array}{l}\text { Mide la proporción que significan los intereses pagados en } \\
\text { relación los recursos obtenidos por la operación. }\end{array}$ \\
\hline Petróleo & $\begin{array}{l}\text { Precio del barril } \\
\text { de petróleo }\end{array}$ & Valor en dólares por barril de petróleo. \\
\hline PIB & $\begin{array}{l}\text { Producto Interno } \\
\text { Bruto }\end{array}$ & Bienes y servicios producidos en el país. \\
\hline
\end{tabular}




\begin{tabular}{|c|c|c|}
\hline Variable & Construcción & Descripción \\
\hline $\begin{array}{l}\text { RC } \\
\text { Razón circulante }\end{array}$ & $\begin{array}{l}\text { Activos } \\
\text { Circulantes } \div \\
\text { Pasivo Circulante }\end{array}$ & $\begin{array}{l}\text { Veces que se pueden pagar las deudas de corto plazo con } \\
\text { los activos de corto plazo. }\end{array}$ \\
\hline Prime Rate & $\begin{array}{l}\text { Tasa de interés } \\
\text { preferencial en } \\
\text { EUA }\end{array}$ & $\begin{array}{l}\text { Registra la tasa de interés que se cobra en EEUU por } \\
\text { financiarse. }\end{array}$ \\
\hline $\begin{array}{l}\text { PT/CC } \\
\text { Pasivo total a } \\
\text { Capital Contable }\end{array}$ & $\begin{array}{l}\text { Pasivo Total } \div \\
\text { Capital Contable }\end{array}$ & $\begin{array}{l}\text { Refleja la cantidad de deudas frente aportación de los } \\
\text { socios. }\end{array}$ \\
\hline $\begin{array}{l}\text { PT/AT } \\
\text { Apalancamiento }\end{array}$ & $\begin{array}{l}\text { Pasivo Total } \div \\
\text { Activo Total }\end{array}$ & $\begin{array}{l}\text { Registra el nivel de uso de deudas con relación a la } \\
\text { inversión total. }\end{array}$ \\
\hline $\begin{array}{l}\text { PME/VN } \\
\text { Pasivo Moneda } \\
\text { extranjera }\end{array}$ & $\begin{array}{l}\text { Pasivo Moneda } \\
\text { Extranjera } \div \\
\text { Ventas Netas }\end{array}$ & $\begin{array}{l}\text { Mide la cantidad de deudas en moneda extranjera en } \\
\text { términos de ventas netas. }\end{array}$ \\
\hline $\begin{array}{l}\text { UB/VT } \\
\text { Margen Utilidad } \\
\text { Bruta }\end{array}$ & $\begin{array}{l}\text { Utilidad Bruta } \div \\
\text { Ventas Totales }\end{array}$ & $\begin{array}{l}\text { Registra el margen de utilidad bruta en términos de } \\
\text { ventas. }\end{array}$ \\
\hline $\begin{array}{l}\text { UN/VT } \\
\text { Margen utilidad } \\
\text { Neta }\end{array}$ & $\begin{array}{l}\text { Utilidad Neta } \div \\
\text { Ventas Totales }\end{array}$ & Registra el margen de utilidad neta en términos de ventas. \\
\hline $\begin{array}{l}\text { UN/AT } \\
\text { Rendimiento del } \\
\text { Activo }\end{array}$ & $\begin{array}{l}\text { Utilidad Neta } \div \\
\text { Activo Total }\end{array}$ & Cuantifica el beneficio de la inversión total. \\
\hline $\begin{array}{l}\text { UN/CC } \\
\text { Rendimiento del } \\
\text { Capital }\end{array}$ & $\begin{array}{l}\text { Utilidad Neta } \div \\
\text { Capital Contable }\end{array}$ & Cuantifica el beneficio de la inversión de los accionistas. \\
\hline $\begin{array}{l}\text { VN/AT } \\
\text { Ventas Netas }\end{array}$ & $\begin{array}{l}\text { Ventas netas } \div \\
\text { Activo total }\end{array}$ & $\begin{array}{l}\text { Porcentaje de ventas netas en relación con la inversión } \\
\text { total. }\end{array}$ \\
\hline $\begin{array}{l}\text { VN/CTN } \\
\text { Cobertura de } \\
\text { Capital Trabajo } \\
\text { Neto }\end{array}$ & $\begin{array}{l}\text { Ventas Netas } \div \\
\text { Capital Trabajo } \\
\text { Neto }\end{array}$ & $\begin{array}{l}\text { Refleja las veces que las ventas representan la inversión en } \\
\text { activos circulantes ocupados para la operación. }\end{array}$ \\
\hline
\end{tabular}

Fuente: elaboración con cálculos propios basados en las definiciones de las NIF (2018).

Es importante considerar que las empresas analizadas pueden presentar un alto grado de heterogeneidad, por ejemplo, diferencias causadas por particularidades debido a la escala de las empresas muestreadas, así como atribuibles a los sectores a los que éstas pertenecen, razón por la cual se optó, como lo sugiere la literatura (por ejemplo, Baltagi, 2005), por recurrir a los modelos econométricos para datos organizados en panel. 
La especificación general del modelo es:

$$
y_{i t}=\alpha+X^{\prime} \beta+\varepsilon_{i t}
$$

donde, $y_{i t}$ es la proporción de las ganancias atribuibles al saldo cambiario (SCUN) de la empresa $i$ en el periodo $t, \alpha$ es el intercepto, $X$ es una matriz que en nuestro caso contiene a las variables explicativas internas de la empresa y macroeconómicas, $\beta$ es un vector de pendientes y $\varepsilon_{i t}$ es un término no observable de perturbación aleatoria. Un problema que puede presentarse con esta especificación es la existencia de sesgos por no considerar las posibles características específicas de las empresas dada la heterogeneidad presente en la muestra analizada y que no se pueden observar en forma directa, como son los efectos de las decisiones que pudieron tomar distintas empresas ante un mismo escenario. Una forma de dar cabida a esas características específicas no observables podría ser el suponer que el término de perturbación puede tener la forma:

$$
\varepsilon_{i t}=\mu_{i}+u_{i t}
$$

es decir, el término de perturbación aleatoria puede estar compuesto por un efecto atribuible a la empresa o específico $\mu_{i}$ y un efecto totalmente aleatorio $u_{i t}$. Si se considera que el efecto específico de la empresa es no aleatorio, se tiene el modelo de efectos fijos:

$$
\begin{gathered}
y_{i t}=\alpha_{i}+X^{\prime} \beta+u_{i t}, \\
\alpha_{i}=\alpha+\mu_{i} .
\end{gathered}
$$

Es decir, en lugar del intercepto general como en (1) ahora se permite que éste cambie de empresa a empresa, manteniéndose constante los valores de los parámetros de las pendientes contenidas en el vector $\beta$. Si se considera que el efecto individual es de naturaleza aleatoria se tiene el modelo de efectos aleatorios:

$$
\begin{aligned}
& y_{i t}=\alpha+X^{\prime} \beta+\varepsilon_{i t} \\
& \varepsilon_{i t}=v_{i}+u_{i t} .
\end{aligned}
$$

Una ventaja importante del modelo de efectos aleatorios es que requiere la estimación de un menor número de parámetros, reduciendo la pérdida de grados de libertad, lo que a su vez permite la estimación de parámetros con menores errores estándar.

Es importante considerar que, en lugar de efectos específicos y constantes atribuibles a las empresas, podría haber en su lugar efectos constantes atribuibles al tiempo, incluso podrían concurrir tanto efectos derivados de la heterogeneidad de las empresas como de los periodos analizados, lo que implicaría la necesidad de incluirlos en las ecuaciones (3) o (4), dependiendo si son o no aleatorios. 


\section{Análisis econométrico}

En la Figura 1 se observa que el SCUN promedio, considerando las 84 empresas estudiadas, fue predominantemente negativo de 1990 a 1995, lo que sugiere pérdidas cambiarias importantes para muchas empresas y que en la parte final de ese periodo puede explicarse como resultado de que la crisis de 1995, como señalan Watkins, Spronk y Félix (2005), aumentó el valor en dólares de la deuda que empresas mexicanas contrataron en esa moneda. Las fugas de capital atribuibles a las tensiones políticas durante 1994 y el crecimiento del déficit de cuenta corriente, a razón de un punto porcentual anual en promedio de 1989 a1994, fueron presiones cambiarias muy importantes. Entre fines de 1997 y principios de 1999 se observan alzas bruscas, seguidas por varios años de relativa calma, hasta que entre 2002 y 2004 se observan nuevamente altibajos notables, indicando que las empresas estudiadas obtuvieron ganancias cambiarias, pese a la crisis asiática de 1997. Es conveniente destacar que, según el INEGI, el PIB mexicano del tercer trimestre de 1994 alcanzó los 520 mil millones de dólares y descendió a 287 mil millones de dólares en el primer trimestre de 1995, es decir, se contrajo 44\%, y logró recuperarse hasta el primer trimestre de 1999 cuando ascendió a 547 mil millones de dólares. Entre 2005 y 2013 el SCUN muestra bastante estabilidad, sin embargo, después regresó a un nivel de valores negativos.

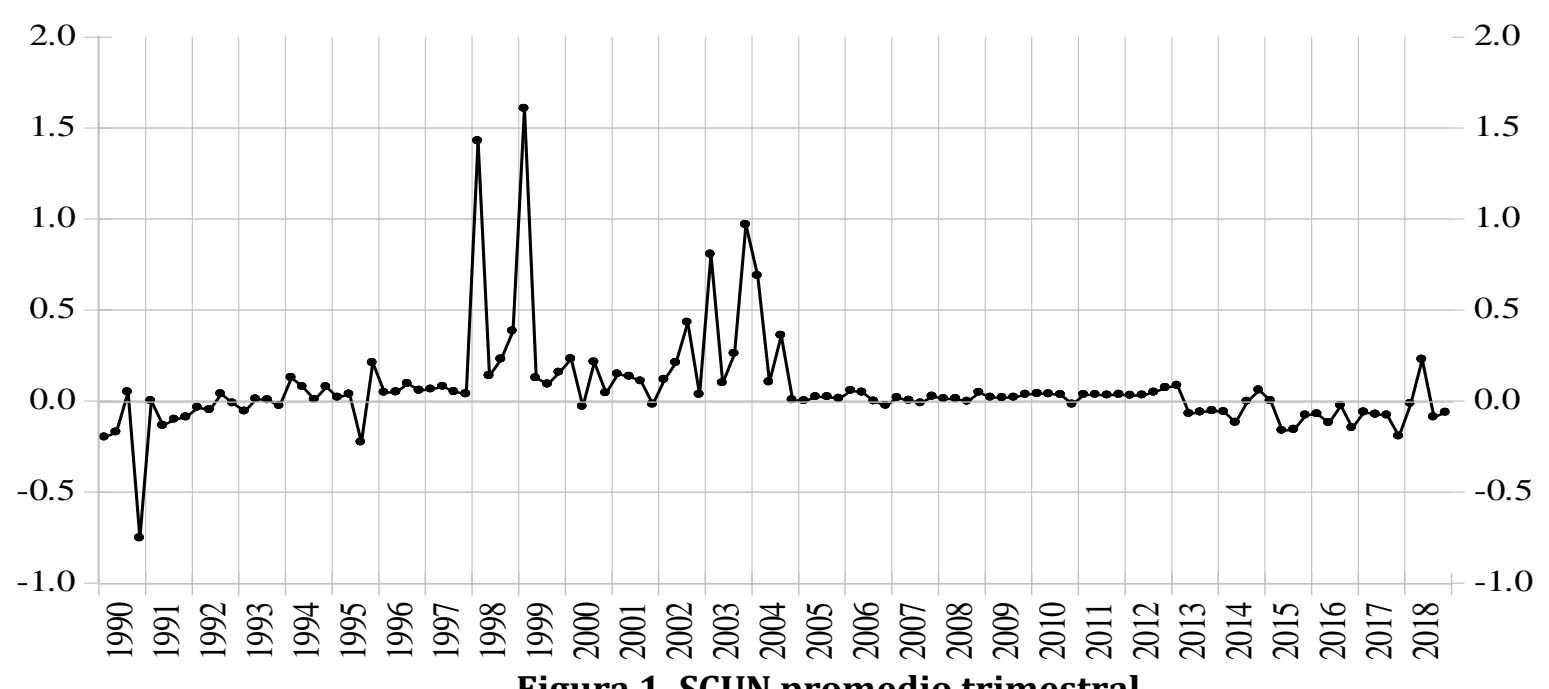

Figura 1. SCUN promedio trimestral

Fuente: elaboración con cálculos propios

En el Cuadro 2 se observa que para cada año las empresas que reportaron SCUN negativos y positivos pertenecen a diferentes sectores. Durante la década de 1990 las empresas con SCUN más altos fueron predominantemente de los sectores de la construcción e industrial; para el período de 2000 a 2010 del sector minero y del de telecomunicaciones. Los sectores que reportaron SCUN más negativos durante la década de 1990 fueron las empresas de los sectores industrial, materiales y consumo frecuente. En el sector industrial se encontró que GMD en el primer trimestre de 1996 tuvo el SCUN más alto y en el siguiente trimestre el más bajo. Para el periodo de 2000 a 2010 fueron en su 
mayoría empresas del sector materiales, y en menor medida del de consumo no básico, industrial y telecomunicaciones; entre 2010 y 2018 del industrial y telecomunicaciones. Además, se observa que los SCUN máximos y mínimos se presentaron durante el período de 1998 a 2004, siendo excepción AMX quien a fines de 2018 obtuvo un SCUN positivo comparable al de ese periodo, en tanto que ICH muestra a principios de 1990 el SCUN más desfavorable de todo el horizonte de estudio.

Cuadro 2. Empresas con SCUN promedio mínimo y máximo por año (\% de la ganancia promedio total)

\begin{tabular}{|l|l|l|r|l|l|l|l|l|l|}
\hline Año & Mínimo & Empresa & Máximo & Empresa & Año & Mínimo & Empresa & Máximo & Empresa \\
\hline 1990 & -20.6584 & ICH & 5.7587 & ICH & 2005 & -0.6769 & PINFRA & 1.3394 & QUMMA \\
\hline 1991 & -0.9023 & REALTUR & 1.8350 & ICA & 2006 & -2.5345 & QUMMA & 4.0431 & HILASAL \\
\hline 1992 & -2.0684 & AHMSA & 1.6393 & GCC & 2007 & -1.3503 & DINE & 1.7700 & GRUMA \\
\hline 1993 & -1.4328 & AHMSA & 0.2647 & REALTUR & 2008 & -1.5608 & PEÑOLES & 1.4847 & POCHTEC \\
\hline 1994 & -1.1104 & HERDEZ & 5.1277 & GMD & 2009 & -1.2947 & GFAMSA & 1.1548 & AUTLAN \\
\hline 1995 & -8.2388 & SIMEC & 6.6381 & CMOCTEZ & 2010 & -0.6934 & ACCESAL & 1.3578 & MFRISCO \\
\hline 1996 & -3.3095 & GMD & 1.0041 & GMD & 2011 & -1.0594 & ICA & 1.9631 & MFRISCO \\
\hline 1997 & -1.1814 & MINSA & 1.3819 & AZTECA & 2012 & -0.9120 & CERAMIC & 2.7283 & MFRISCO \\
\hline 1998 & -8.7695 & HERDEZ & 77.3077 & AHMSA & 2013 & -7.4830 & AUTLAN & 4.1926 & MFRISCO \\
\hline 1999 & -7.0730 & GRUMA & 76.9083 & GRUMA & 2014 & -6.6474 & AUTLAN & 5.5835 & GMD \\
\hline 2000 & -12.7475 & GRUMA & 9.0909 & GMD & 2015 & -4.3976 & AMX & 3.2066 & AUTLAN \\
\hline 2001 & -14.6998 & QBINDUS & 9.7768 & GRUMA & 2016 & -8.9333 & AUTLAN & 8.8139 & COLLADO \\
\hline 2002 & -18.1949 & SIMEC & 17.1353 & PEÑOLES & 2017 & -5.4193 & CONVER & 3.0722 & CIE \\
\hline 2003 & -12.5867 & PEÑOLES & 58.5358 & HILASAL & 2018 & -7.0637 & ALFA & 29.0988 & AMX \\
\hline 2004 & -5.6167 & HILASAL & 32.500 & HILASAL & & & & & \\
\hline
\end{tabular}

Fuente: elaboración con cálculos propios

En el Cuadro 3 se muestran los resultados del modelo estimado por mínimos cuadrados ordinarios y de los modelos de efectos fijos y efectos aleatorios estimados, modelos base del análisis. La especificación con efectos aleatorios se llevó a cabo mediante el estimador EGLS factible y no sesgado de Swamy-Arora, estimador que permite relajar los supuestos de heteroscedasticidad y de no autocorrelación entre periodos de tiempo considerados en el método de mínimos cuadrados ordinarios; para tomar en cuenta la posible correlación contemporánea entre las empresas y la heteroscedasticidad se consideró también una matriz robusta de covarianzas de los coeficientes estimados. Grosso modo, las estimaciones muestran que las tres especificaciones del modelo coinciden en la mayor parte de coeficientes que resultaron significativos incluyendo sus signos, sin embargo, sus magnitudes difieren relativamente. 
Cuadro 3. Estimaciones de los modelos con todas las variables

\begin{tabular}{|c|c|c|c|c|c|c|}
\hline \multicolumn{7}{|c|}{ Variable dependiente: SCUN } \\
\hline Variable & \multicolumn{2}{|l|}{ MCO } & \multirow{2}{*}{\begin{tabular}{|r|} 
Efectos fijos \\
0.743018
\end{tabular}} & \multirow[b]{2}{*}{$* * *$} & \multicolumn{2}{|c|}{ Efectos aleatorio } \\
\hline Constante & 0.446871 & *** & & & 0.570062 & *** \\
\hline$(\mathrm{AC}-\mathrm{INV}) / \mathrm{PC}$ & -0.025895 & ** & -0.002562 & & -0.012496 & \\
\hline $\mathrm{AC} / \mathrm{AT}$ & -0.074585 & & -0.115401 & & -0.081559 & \\
\hline $\mathrm{AI}$ & 0.122577 & & 0.469004 & *** & 0.313562 & *** \\
\hline AF/Act_Inv & $-5.13 E-05$ & *** & $-4.13 \mathrm{E}-05$ & *** & $-4.37 \mathrm{E}-05$ & *** \\
\hline CTN & 0.088743 & *** & 0.063189 & & 0.052409 & \\
\hline CETES & -0.001471 & & -0.001697 & & -0.001541 & \\
\hline DME/AT & 0.697821 & **** & 0.556162 & & 0.610054 & \\
\hline DME/PT & $6.16 \mathrm{E}-05$ & & $2.87 \mathrm{E}-04$ & & 0.000258 & \\
\hline Dólar & -1.379634 & *** & -1.598884 & **** & -1.507306 & *** \\
\hline $\mathrm{E} / \mathrm{PC}$ & 0.009427 & & 0.021841 & & 0.014639 & \\
\hline FA/TF & -0.002158 & & -0.00349 & & -0.003082 & \\
\hline $\mathrm{FP} / \mathrm{TF}$ & -0.002419 & & -0.003996 & & -0.003598 & \\
\hline FCT/VN & 0.003317 & & 0.00236 & & 0.0028 & \\
\hline FRN/VN & -0.001926 & & -0.002584 & & -0.002505 & \\
\hline $\mathrm{AF} / \mathrm{AT}$ & 0.086878 & & -0.096601 & & 0.009227 & \\
\hline I/RGO & 0.000501 & & 0.000287 & & 0.000279 & \\
\hline Petróleo & -0.000797 & ** & -0.000781 & * & -0.00081 & ** \\
\hline PIB & $-2.14 \mathrm{E}-14$ & *** & $-2.71 \mathrm{E}-14$ & **** & $-2.45 \mathrm{E}-14$ & *** \\
\hline $\mathrm{RC}$ & -0.051125 & * & -0.041724 & & -0.023602 & \\
\hline Prime Rate & -0.006917 & & -0.00525 & & -0.0062 & \\
\hline $\mathrm{PT} / \mathrm{CC}$ & -0.00197 & & -0.002036 & & -0.002006 & \\
\hline $\mathrm{PT} / \mathrm{AT}$ & 0.036196 & & -0.038343 & & -0.01177 & \\
\hline PME/VN & $-1.86 \mathrm{E}-06$ & & $-5.69 \mathrm{E}-05$ & & $-7.48 \mathrm{E}-05$ & \\
\hline UB/VT & 0.000644 & & 0.002598 & & 0.000833 & \\
\hline \begin{tabular}{|l|}
$\mathrm{UN} / \mathrm{AT}$ \\
\end{tabular} & -0.001309 & & -0.001953 & & -0.000948 & \\
\hline UN/AT & 0.023988 & & -0.000655 & & -0.004264 & \\
\hline UN/CC & 0.004002 & & 0.001232 & & 0.002562 & \\
\hline VN/AT & -0.01133 & & -0.146606 & ** & -0.073757 & ** \\
\hline VN/CTN & 0.000509 & & 0.000578 & & 0.000578 & \\
\hline$F$ & 1.863759 & *** & 3.046327 & *** & 1.444834 & $* * *$ \\
\hline
\end{tabular}

Fuente: elaboración propia con nuestras estimaciones

En el Cuadro 4 se muestran las diversas pruebas de especificación, iniciando por las pruebas de existencia de efectos de heterogeneidad no observada, con base en sus resultados se puede 
deducir que el modelo adecuado debería incluir efectos individuales de la empresa, pero no efectos temporales no observados, destacando al respecto la coincidencia de las pruebas diseñadas específicamente para paneles no balanceados como es el caso del que se emplea en este análisis. La prueba de razón de verosimilitud sugiere que los efectos fijos estimados mediante mínimos cuadrados son significativos. Por su parte, la prueba de Hausman sugiere no rechazar la hipótesis nula de ausencia de correlación entre los efectos y los regresores, por lo que tanto las estimaciones de efectos aleatorios como de efectos fijos son consistentes, sin embargo, bajo esa hipótesis nula los parámetros del modelo de efectos fijos son ineficientes (tienen mayor varianza), razón por la cual sería preferible considerar los efectos individuales de las empresas como aleatorios.

Cuadro 4. Pruebas de especificación para el modelo con todas las variables

\begin{tabular}{|c|c|c|c|c|c|c|}
\hline \multicolumn{4}{|c|}{$H_{0}$ : No hay efectos de heterogeneidad no observada } & \multirow{2}{*}{\multicolumn{3}{|c|}{$\begin{array}{c}H_{0} \text { : Efectos fijos no significativos } \\
\text { Razón de verosimilitud }\end{array}$}} \\
\hline & Empresas & & Tiempo & & & \\
\hline Breusch-Pagan & 292.5439 & *** & 1.4396 & $F$ & 3.4513 & *** \\
\hline Honda & 17.1039 & *** & -1.1999 & $c^{2}$ & 278.4746 & *** \\
\hline King-Wu & 17.1039 & *** & -1.1999 & \multicolumn{3}{|c|}{ Efectos fijos vs aleatorios } \\
\hline Honda estandarizada & 18.4656 & *** & -0.8260 & \multicolumn{3}{|c|}{ Prueba de Hausman } \\
\hline King-Wu estandarizada & 18.4656 & *** & -0.8260 & $c^{2}$ & 6.3967 & \\
\hline
\end{tabular}

Los coeficientes de la mayor parte de las variables del modelo con efectos aleatorios no son significativos, por lo que se procedió a reducir el modelo retirando en forma secuencial bloques de variables cuyo contenido informativo está teóricamente contenido en otras variables incluidas en el modelo, buscando en cada paso el valor máximo local del estadístico $F$ que, como puede verse en el Cuadro 5 se alcanza en el sexto paso de reducción cuando dicho estadístico alcanza un máximo local después del cual desciende.

Cuadro 5. Pasos de reducción del modelo de efectos aleatorios

\begin{tabular}{|c|l|c|}
\hline Paso & Variables restringidas & $\boldsymbol{F}$ \\
\hline 0 & Ninguna & 1.444834 \\
\hline 1 & $\begin{array}{l}\text { AF/AT, I/RGO, RC, Prime Rate, PT/AT, PME/VN, } \\
\text { UB/VT, UN/VT, UN/AT y UN/CC }\end{array}$ & 2.464819 \\
\hline 2 & DME/PT, E/PC, FA/TF, FP/TF & 3.186437 \\
\hline 3 & CETES & 3.411980 \\
\hline 4 & FA/FT & 3.720901 \\
\hline 5 & PETROLEO, VN/CTN & 4.473439 \\
\hline 6 & FRN/VN & 5.028027 \\
\hline 7 & DME/AT & 4.114442 \\
\hline
\end{tabular}


El modelo restringido se presenta en el cuadro 6, donde se observa que la prueba de restricciones de todos los parámetros retirados del modelo de efectos aleatorios del Cuadro 3 alcanza un valor cercano a 0.1115 , impidiendo rechazar la hipótesis nula de que los parámetros retirados no son conjuntamente significativos, apoyando la decisión de retirar las variables que quedaron fuera del modelo mediante el proceso de reducción.

Cuadro 6. Modelo restringido con efectos aleatorios

\begin{tabular}{|c|c|c|}
\hline \multicolumn{3}{|c|}{ Variable dependiente: SCUN } \\
\hline Variable & Coeficiente & \\
\hline Constante & 0.417685 & *** \\
\hline $\mathrm{AC} / \mathrm{AT}$ & -0.097893 & 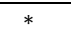 \\
\hline $\mathrm{AI}$ & 0.241361 & *** \\
\hline AF/Act_Inv & $-4.20 \mathrm{E}-05$ & $* * *$ \\
\hline CTN & 0.023226 & *** \\
\hline $\mathrm{DME} / \mathrm{AT}$ & 0.598904 & $*$ \\
\hline Dólar & -1.383463 & *** \\
\hline PIB & $-2.30 \mathrm{E}-14$ & $* * *$ \\
\hline VN/AT & -0.062951 & ** \\
\hline$F$ & 5.028027 & $* * *$ \\
\hline \multicolumn{3}{|c|}{ Prueba de restricciones ${ }^{a}$} \\
\hline$F$ & 0.1114999 & \\
\hline \multicolumn{3}{|c|}{$\begin{array}{l}\text { ***, }{ }^{* *} \mathrm{y}^{*} \text { denotan, respectivamente el } \\
1 \text {, el } 5 \text { y el } 10 \% \text { de significancia } \\
\text { a restringiendo el valor de los parámetros retirados a cero }\end{array}$} \\
\hline
\end{tabular}

Fuente: elaboración propia con nuestras estimaciones

El cuadro 7 muestra las pruebas de especificación correspondientes al modelo reducido que, al igual que con el modelo completo sugiere que, dada la existencia de efectos de heterogeneidad de las empresas analizadas, la especificación del modelo reducido con efectos aleatorios parece preferible que la de efectos fijos.

Cuadro 7. Pruebas de especificación del modelo restringido

\begin{tabular}{|c|c|c|c|c|c|c|}
\hline \multicolumn{4}{|c|}{$\begin{array}{l}H_{0}: \text { No hay efectos de heterogeneidad no } \\
\text { observada }\end{array}$} & \multirow{2}{*}{\multicolumn{3}{|c|}{$\begin{array}{c}H_{0}: \text { Efectos fijos no significativos } \\
\text { Razón de verosimilitud } \\
\end{array}$}} \\
\hline & Empresas & & Tiempo & & & \\
\hline Breusch-Pagan & 304.2854 & *** & 1.3888 & $F$ & 3.4936 & $* * *$ \\
\hline Honda & 17.44378 & *** & -1.1785 & $c^{2}$ & 284.4748 & *** \\
\hline King-Wu & 17.44378 & *** & -1.1785 & \multicolumn{3}{|c|}{ Efectos fijos vs aleatorios } \\
\hline Honda estandarizada & 18.29961 & *** & -1.0022 & \multicolumn{3}{|c|}{ Prueba de Hausman } \\
\hline
\end{tabular}




\begin{tabular}{|c|c|c|c|c|c|c|}
\hline $\begin{array}{l}\text { King-Wu } \\
\text { estandarizada }\end{array}$ & 18.29961 & *** & -1.0022 & $c^{2}$ & 5.4180 & \\
\hline$* * *=1 \%$ de sig & & & & & & \\
\hline
\end{tabular}

Aunque poco significativa la inversión en activos corrientes, su signo muestra que los incrementos de ese tipo de inversión han disminuido la importancia que tienen las ganancias cambiarias en las ganancias totales de las empresas, pudiendo contribuir a la explicación de los casos de pérdidas cambiarias. El signo del coeficiente de la inversión en activos intangibles sugiere que ésta ha contribuido al logro de un mayor peso de las ganancias cambiarias en los resultados de las empresas. Aunque muy pequeño en magnitud, el coeficiente de la inversión a largo plazo indica que ésta ha propiciado disminuciones en la importancia del saldo cambiario dentro de las ganancias totales. Los resultados obtenidos en relación con la importancia de las inversiones en las ganancias cambiarias están en línea con los hallazgos de Desai, Foley y Forbes (2007) quienes encontraron que las empresas multinacionales estadounidenses expandieron sus ventas, los activos e inversión durante las fuertes depreciaciones. En cuanto al capital de trabajo, se observa que ha contribuido a incrementar la participación del saldo cambiario en los beneficios contables durante el periodo de análisis.

Por su parte, es poco significativa la deuda en moneda extranjera respecto de la inversión total, aunque el signo sugiere que su incremento puede aumentar la ponderación que tienen las ganancias del saldo cambiario, nuestros resultados son distintos a los hallazgos de Kim, Tesar y Zhang (2015) y Keysriyeli, Özmen y Yigit. (2011) quienes evidenciaron que la deuda en moneda extranjera afectó negativamente las inversiones y ganancias empresariales durante las depreciaciones monetarias, lo que sugiere que las empresas mexicanas utilizaron el financiamiento para ampliar su capacidad de producción de productos de exportación. Cuando ocurre una depreciación de la moneda doméstica, la deuda en moneda extranjera aumenta, por una parte, se incrementan el monto del principal por el encarecimiento del dólar, y por la otra los intereses también aumentan por que se pagan en dólares, estos ajustes se reflejan en el saldó de las pérdidas cambiarias.

El dólar muestra una influencia negativa en dicha ponderación, siendo el coeficiente que más impacto tiene por su magnitud. Aunque de magnitud pequeña, la influencia del PIB ha sido negativa para la contribución que hacen las ganancias cambiarias al resultado total, pudiendo deberse a la pérdida sostenida del peso frente al dólar observada durante el periodo de análisis. El efecto de estas variables reflejó el mismo efecto que los hallazgos de Bonono, Martins y Pinto (2003) en las empresas de Brasil. Por último, se observa que las ventas en relación con el activo total se relacionan negativamente con el peso de las ganancias cambiarias.

\section{Conclusiones}

Mediante modelos para datos en panel se estudiaron las ganancias cambiarias de 1990 a 2018 en una muestra de empresas mexicanas, logrando el objetivo de identificar variables económicas y fundamentales que inciden en la determinación de las ganancias cambiarias como consecuencia de 
los saldos cambiarios corporativos. De manera congruente con la literatura previa, las variables económicas que resultaron significativas son el dólar y el PIB. Las variables fundamentales significativas son la inversión en activos fijos, la deuda en moneda extranjera, la inversión en activos intangibles, las ventas sobre el activo total, la inversión en activos corrientes y el capital de trabajo neto; las últimas cuatro no habían sido estudiadas en la literatura previa.

Como sugerencias generales para los tomadores de decisiones empresariales, se puede decir que es recomendable mantener al mínimo posible los niveles de la inversión en capital de trabajo y en activos corrientes para incrementar su flujo de efectivo y así se reduzca la posibilidad de crear pérdidas cambiarias. Como podría anticiparse, es importante el desarrollo de estrategias para incrementar las ventas en moneda extranjera con el objetivo de reducir la posibilidad de pérdidas cambiarias. Las decisiones de inversión en maquinaria, planta, equipo y activos intangibles denominados en moneda extranjera deben hacerse considerando su incidencia en los descalces cambiarios y, en última instancia, en los resultados finales vía las ganancias y/o pérdidas potenciales derivadas de esos descalces. La comprensión de las variables que afectan las ganancias cambiarias también puede ser de utilidad en términos de la administración de los riesgos financieros de la empresa.

Si bien en este trabajo se ha proporcionado evidencia sobre la importancia de variables económicas y fundamentales en el peso que tienen los saldos cambiarios en las ganancias cambiarias corporativas, es importante tener presente las diferencias no triviales entre las empresas debido al sector de actividades y a sus peculiaridades individuales, por ejemplo su papel preponderante como importadoras o exportadoras, que pueden condicionar las operaciones y las oportunidades y necesidades de inversión y financiamiento.

\section{Referencias}

[1] A.C.-CINIF. (2018). Consejo Mexicano para la Investigación y Desarrollo de Normas de Información Financiera. NIF A-3. CINIF-IMCP, México.

[2] Aghion, P.; Bacchetta, P. y Banerjee, A. (2000). Currency Crises and Monetary Policy in an Economy whit Credit Constraints. Third draft. doi: http://dx.doi.org/10. 2139/ssrn.239288

[3] Aguiar, M. (2005). Investment, Devaluation, and Foreign Currency Exposure: The Case of México, Journal of Development Economics, 78 (1), 95-113. Retrieved from: https://www.sciencedirect.com/science/article/abs/pii/S0304387805000398?via\%3Dihub

[4] Allayannis, G.; Brown, G.; y Klapper, L. (2003). Capital structure and financial risk: evidence from foreign debt use in East Asia. The Journal of Finance, 58 (6), 2667-2710. doi: https://doi.org/10.1046/j.1540-6261.2003.00619.x

[5] Bae, S.; Kim H. y Kwon, T. (2018). Foreign currency debt financing, firm value, and risk: Evidence from Korea surrounding the global financial crisis. Asia-Pacific Journal of Financial Studies, 45(1), 124-152. doi: https://onlinelibrary.wiley.com/doi/abs/10.1046/j.1540-6261.2003. 00619.x

[6] Bae, S.; Kwon, T. \& Lee, J. (2011). Does corporate diversification by business groups create value? Evidence from Korea Chaebols. Pacific-Basin Finance Journal, 19(5), 535-553. doi: dhttps://doi.org/10.1016/j.pacfin.2011.04.001

[7] Baltagi, B. H. (2005). Econometric analysis of panel data. John Wiley \& Sons 3rd ed., Wiltshire. 
[8] Baltagi, B. H., Chang, Y. J., y Q. Li (1992). Monte Carlo results on several new and existing tests for the error components model. Journal of Econometrics, 54 (1-3), 95-120. doi: https://doi.org/10.1016/0304-4076(92)90101-v

[9] Baltagi, B. H., Chang, Y. J., y Q. Li (1999). Testing for random individual and time effects using unbalanced panel data. Advances in Econometrics, 13, 1-20. doi: https://doi.org/10.1108/s07319053(1999)0000013003

[10] Banco de México, https://www.banxico.org.mx

[11] Benavente, J., M.; Johnson, C. A. y G. Morandé, F. G. (2003). Debt composition and balance sheet effects of exchange rate depreciations: a firm-level analysis for Chile. Emerging Markets Review, 4,397-416. doi: https://doi.org/10.1016/s1566-0141(03)00062-1

[12] Bonono, M.; Martins, B. y Pinto R. (2004). Debt composition and exchange rate balance sheet effects in Brazil: a firm level analysis. Ensaios Econômicos, No. 535, 1-39.

[13] Brown, M.; Ongean, S. y Yesin, P. (2011). Foreign Currency Borrowing by Small Firms in the Transition Economies. J. Finan. Intermediation, 20,285-302. doi. https://doi.org/10.1016/s15660141(03)00061-x

[14] Caballero, J.; Panizza, U. y Powell, A. (2014). Balance sheets and credit growth. In A. Powell (Ed.), Global Recovery and Monetary Normalization. Escaping Chronicle Foretold? (chap. 4). Inter-American Development Bank.

[15] Céspedes, L. F, Chang, R. y Velasco, A. (2000). Balance sheets and Exchange rate policy. NBER Working Paper No. 7840,1-50. doi: https://doi.org/10.3386/w7840

[16] Céspedes, L.; Chang, R. y Velasco, A. (2004). Balance sheets and Exchange rate policy. American Economic Review, 94(4), 1183-1193. doi: https://doi.org/10.1257/000282804 2002589

[17] Chow, J. (2015). Stress testing Corporate Balance Sheets in emerging economies. International Monetary Fund Working Paper WP/15/215, 1- 18. doi: https://doi.org/10.5089/9781513502717.001

[18] Chui, M., Fender, I., \& Sushko, V. (2014). Risk related to EME corporate balance sheets: the role of leverage and currency mismatch. BIS Working Quarterly Review, September 2014.

[19] Chui, M.; Kuruc, E. y Turner, P. (2016). A new dimension to currency mismatches in the emerging markets: non-financial companies. BIS Working Papers, 550.

[20] Cowan, K.; Hansen E.; y Herrera L.O. (2005). Descalces cambiarios en firmas chilenas no Financieras. Economía Chilena 8, (2), 57-82. Recuperado en: https://si2.bcentral.cl/ public/pdf/revistaeconomia/2005/ago/Vol8N2ago2005pp57_82.pdf

[21] Desai, M.; Foley, C. F. y Forbes, K. (2007). Financial constraints and growth: multinational and local firm responses to currency depreciations. Review of Financial Studies, 21 (6), 2859-2888. doi: https://doi.org/10.1093/rfs/hhm017

[22] Díez, Antonio y Ortiz, A. (2001) Crisis cambiarias en Latinoamérica: factores específicos e internacionales. ICE La Nueva Agenda de América Latina. Núm. 790, 93-106. Recuperado en: https://www.researchgate.net/publication/28050982_Crisis_c ambiarias_en_Latinoamerica_factores_especificos_e_internacionales

[23] Du, W. y Schreger, J. (2016) Sovereign risk, currency risk, and corporate balance sheets. Working Paper, mimeo. doi: https://doi.org/10.2139/ssrn.2845428

[24] ECONOMATICA, base de datos de empresas que cotizan en las Bolsas de Valores en EUA y América Latina.

[25] Forbes, K. (2002). How do large depreciations affect firm performance? NBER Working Paper 9095. doi: https://doi.org/10.3386/w9095

[26] Frank, M. y Goyal, V. (2003). Testing the pecking order Theory of Capital Structure. Journal of Financial Economics, 67,217-248. doi: https://doi.org/10.2139/ssrn.243138 
[27] García, J.; García, P. y Domenge, R. (2011). Determinantes de la estructura de capital en la pequeña y mediana empresa familiar en México. Contaduría y Administración 57 (3), julio-septiembre, 67-96. Recuperado en: http://www.scielo.org.mx/scielo. php?script=sci_arttext\&pid=S018610422012000300004

[28] Guzmán, M.; de la Paz, L. S. y Antonio Cárdenas. (2007). La relación de causalidad entre el índice bursátil mexicano y el tipo de cambio spot. Análisis Económico, 51, XXII, Tercer cuatrimestre. 81-105. Recuperado en: https://www.redalyc.org/pdf/413/4131 1486005.pdf

[29] Hernández, G. y Ríos, H. (2012). Determinantes de la estructura financiera en la industria manufacturera: la industria de alimentos. Análisis Económico, vol. XXVII, número 65, 101-121. Recuperado en: https://www.redalyc.org/articulo.oa?id=41324 594006 https://www.bis.org/publ/qtrpdf/r_qt1409f.pdf

[30] Instituto Nacional de Geografía e Informática (INEGI): https://www.inegi.org.mx

[31] Kesriyeli, M.; Özmen, E. y Yigit, S. (2011) Corporate Sector Liability Dollarization and Exchange Rate Balance Sheets Effect in Turkey. Applied Economics, 43 (30): 4741-4747. doi: https://doi.org/10.1080/00036846.2010.498353

[32] Kim, Y. J.; Tesar, L., y Zhang, J, (2015). The impact of foreign liabilities on small firms: firm level evidence from the Korean crisis. Journal of International Economics, 97, 209-30. doi: https://doi.org/10.1016/j.jinteco.2015.05.006

[33] Krugman, P. (1999). Balance sheets, the transfer problem, and financial crises. International Tax and Public Finance, 6(4), 459-472. doi: https://doi.org/10.3386/w 17756

[34] Luca, A. y Petrova, I. (2008). What drives credit dollarization in transition economies? Journal of Banking \& Finance, 32,858-869. doi: https://doi.org/10.1016/j.jbankfin. 2007.06.003.

[35] Morales, J. (2019). Volatilidad de las empresas descalzadas del sector discrecional de la Bolsa Mexicana de Valores durante las crisis cambiarias. CIMEXUS, Vol. XIV, No.2, 109-126. doi: https://doi.org/10.33110/cimexus140208.

[36] Morales, J. (2020) Análisis de la solvencia de las empresas descalzadas del sector industrial de la Bolsa Mexicana de Valores. Revista Academia y Negocios, Vol. 5 (2), pp. 43-56.

[37] Morales, J., Rojas, M. y Velázquez, G. (2017). Resultados cambiarios y su relación con el grado de internacionalización de las empresas de la Bolsa Mexicana de Valores. Respuestas, Vol. 22, No. 1, 5974. doi: https://doi.org/10.22463/0122820X.820

[38] Nuñez, G.; Perrotini, I. y López-Herrera, F. (2018). Corporate governance and international bond issues by Latin American corporations. CEPAL Review, No. 128, 29-42. doi: https://doi.org/10.18356/841d9d78-en

[39] Petróleos Mexicanos (PEMEX), https://www.pemex.com/Paginas/default.aspx

[40] Serena, M. y Sousa, R. (2017). Does Exchange rate depreciation have contractionary effects on firm level investment? BIS Working Papers, No. 624. doi: https://doi.org/ 10.2139/ssrn.3139024

[41] Shapiro, A. C. (1984). The impact of Taxation on the Currency-Of Denomination Decision for LongTerm Foreign Borrowing and Lending. Journal of International Business Studies, 15(1), 15-25. doi: https://doi.org/10.1057/palgrave.jibs.8490468

[42] Watkins, K; Spronk, J. y Félix L. (2005). Propagación de crisis en las empresas: la experiencia mexicana. Economía mexicana nueva época. XIV, 1, 119-135. Recuperado en: https://www.redalyc.org/pdf/323/32314104.pdf 


\section{Anexo 1. Empresas de la muestra}

\begin{tabular}{|c|c|c|c|c|c|}
\hline Núm. & Clave de cotización & Núm. & \begin{tabular}{|l|}
$\begin{array}{l}\text { Clave de } \\
\text { cotización }\end{array}$ \\
\end{tabular} & Núm. & Clave de cotización \\
\hline \multicolumn{2}{|c|}{ Productos de consumo no básico } & 31 & KUO & 61 & FEMSA \\
\hline 1 & ALSEA & 32 & LAMOSA & 62 & GIGANTE \\
\hline 2 & CIDMEGA & 33 & OMA & 63 & GMODELO \\
\hline 3 & CIE & 34 & PASA & 64 & GRUMA \\
\hline 4 & CMR & 35 & PINFRA & 65 & HERDEZ \\
\hline 5 & EDOARDO & 36 & SARE & 66 & KIMBER \\
\hline 6 & ELEKTRA & 37 & URBI & 67 & KOF \\
\hline 7 & GFAMSA & 38 & VOLAR & 68 & MASECA \\
\hline 8 & GPH & \multicolumn{2}{|c|}{ Materiales } & 69 & MINSA \\
\hline 9 & HCITY & 39 & AHMSA & 71 & WALMEX \\
\hline 10 & HILASAL & 40 & ALPEK & \multicolumn{2}{|r|}{ Salud } \\
\hline 11 & LIVEPOL & 41 & AUTLAN & 72 & BEVIDES \\
\hline 12 & POSADAS & 42 & CEMEX & 73 & FRAGUA \\
\hline 13 & REALTUR & 43 & CMONTEZ & 74 & LAB \\
\hline 14 & VASCONI & 44 & COLLADO & \multicolumn{2}{|c|}{ Telecomunicaciones } \\
\hline \multicolumn{2}{|r|}{ Industrial } & 45 & CONVER & 76 & AMX \\
\hline 15 & ACCELSA & 46 & CYDSASA & 77 & AXTEL \\
\hline 16 & AGUA & 47 & GCC & 78 & AZTECA \\
\hline 17 & ALFA & 48 & GMEXICO & 79 & CABLE \\
\hline 18 & ARA & 49 & ICH & 80 & MAXCOM \\
\hline 19 & ARISTOS & 50 & MEXCHEM & 81 & MEGA \\
\hline 20 & ASUR & 51 & MFRISCO & 82 & QUMMA \\
\hline 21 & CERAMIC & 52 & PEÑOLES & 83 & RCENTRO \\
\hline 22 & DINE & 53 & POCHTEC & 84 & TLEVISA \\
\hline 23 & GCARSO & 54 & QBINDUS & & \\
\hline 25 & GEO & 55 & SIMEC & & \\
\hline 24 & GISSA & \multicolumn{2}{|c|}{ Productos de consumo frecuente } & & \\
\hline 26 & GMD & 56 & AC & & \\
\hline 27 & HOGAR & 57 & BACHOCO & & \\
\hline 28 & HOMEX & 58 & BAFAR & & \\
\hline 29 & ICA & 59 & BIMBO & & \\
\hline 30 & IDEAL & 60 & COMERCI & & \\
\hline
\end{tabular}

Fuente: elaboración propia con datos de la Bolsa Mexicana de Valores 